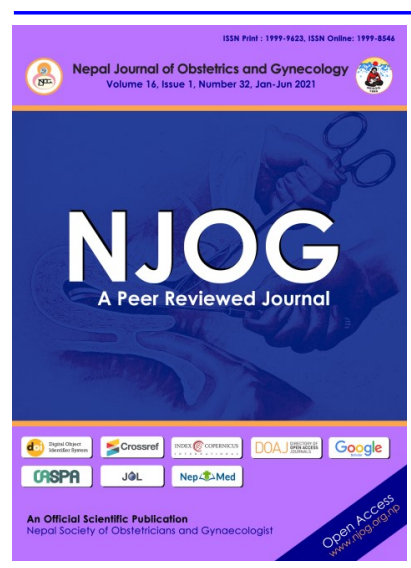

CORRESPONDENCE

Dr Snigdha Rai

Paropakar Maternity and Women's Hospital, Thapathali, Kathmandu, Nepal

\section{Email:}

snigdha_raj@hotmail.com; Phone: +977-9851195477

Received: April 15, 2021 Accepted: May 1, 2021

\section{Citation:}

Rai S. High Grade Endometrial Stromal Sarcoma: a rare case report. Nep J Obstet Gynecol. 2021;16 (32):129-131. DOI: https:// doi.org/10.3126/ njog.v16i1.37621

\title{
High Grade Endometrial Stromal Sarcoma: a rare case report
}

Snigdha Rai

Paropakar Maternity and Women's Hospital, Kathmandu

\section{ABSTRACT}

High grade endometrial stromal sarcoma (HG-ESS) is a very rare entity with poor outcome in compared to other endometrial stromal sarcoma as it is usually diagnosed in late stage. The stage of the disease, age and complete surgery are most important prognostic factors. Surgical management of a 40 years old lady with menometrorrhagia and high grade endometrial stromal sarcoma is presented.

Keywords: endometrial stromal sarcoma, histopathology, surgery

\section{INTRODUCTION}

Uterine sarcomas account about $<1 \%$ of all uterine malignancies and arises from mesodermal structures like muscles and connective tissues. ${ }^{1-3}$ Mesenchymal tumors of the uterus with cytological and architectural features reminiscent of endometrial stromal cells are classified as endometrial stromal tumors. The recent World Health Organization Classification of Tumors of the Breast and Female Genital Organs divides stromal neoplasm into benign endometrial stromal nodule (ESN), Low Grade endometrial stromal sarcoma (LGESS), High Grade Endometrial Stromal Sarcoma (HSESS) and undifferentiated endometrial sarcoma. ${ }^{3}$ High grade endometrial stromal sarcoma is very rare especially among the young population.

\section{CASE}

A 40-year-old para 2 lady with regular previous menstrual cycle came with menometrorrhagia for 3 months and endometrial biopsy report as low grade endometrial stromal neoplasia. She had no significant medical, surgical history and family history. She was nonsmoker and nonalcoholic with contraceptive history of depot medroxyprogesterone acetate 10 years ago. Her general and vital signs were normal. Cervix bled on touch with speculum blade; and uterus and adnexae were normal. MRI revealed endometrial thickness of 9 $\mathrm{mm}$ with fluid in endometrial cavity and low signal nodule about 1.1 $\times 2 \mathrm{~cm}$ in the fundal region of endometrial cavity. [Figure-1]

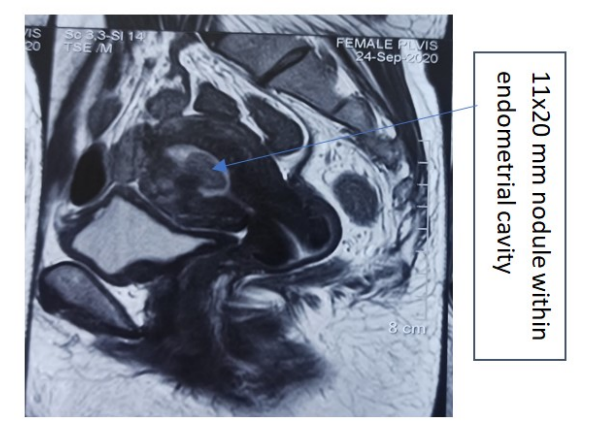

Figure-1: MRI showing single nodule in endometrial cavity

Staging laparotomy with TAH/BSO, lymph node dissection and omental biopsy was performed. Intraoperative findings revealed 8 weeks uterus, right ovarian cyst of $3 \times 3 \mathrm{~cm}$ with bilateral tubes adherent to the posterior surface of the uterus and colon. Lymph nodes and omentum grossly appeared normal. There was a sessile growth at left cornu within uterine cavity posteriorly. Her postoperative period was unremarkable.

Histopathological report confirmed high grade endometrial stromal sarcoma of maximum tumor dimension $4.5 \mathrm{~cm}$ with invasion into upper half of myometrium; lymph nodes and omentum were free of tumor and lymphovasular invasion was not seen. Microscopically, focal areas showed moderate to marked nuclear pleomorphism and mitotic figures of 15/10 high power field along with the areas of necrosis was seen.(Figure-2 and 3) The patient was referred to radiation oncology department and after discussion the patie- 
$\mathrm{nt}$ is kept under regular follow up.

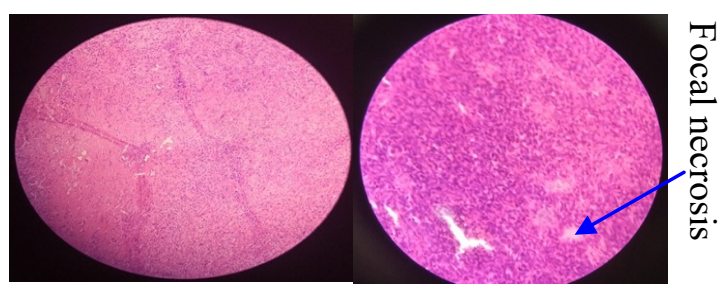

Figure-2: 40x with atypia Figure-3: 40x showing areas of focal necrosis

\section{COMMENTS}

Endometrial stromal sarcoma is very rare malignant tumor contributing approximately $10 \%$ of all uterine sarcomas but only $0.2 \%$ of all uterine malignancy and is usually presented in late stage. ${ }^{2}$ In 2003, the WHO changed the diagnostic criteria after which the term endometrial sarcoma was restricted to an invasive endometrial stromal neoplasm with a distinct histologic appearance with very good prognosis, while the term undifferentiated endometrial stromal sarcoma was introduced for those with more pleomorphic appearance with prominent tumor necrosis and very poor prognosis.

HG-ESS is a rare pathological type of uterine sarcoma. The mean age of ESS ranges between 45 and 55 years however there is a case report of HG-ESS in a patients of 21 years. ${ }^{4,5}$ The patients may present with the common symptoms of abnormal uterine bleeding, pelvic pain and uterine mass or endometrial polyp. ${ }^{5}$ Though stage of the disease, age and complete surgery are mentioned to be most important prognostic factors by some authors, information regarding the natural course, prognostic factors and optimal treatment for HG- ESS is very limited at present. $^{5}$ The disease is often misdiagnosed as uterine leiomyoma or endometrial carcinoma prior to surgery due to lack of characteristics image and clinical manifestations. ${ }^{6}$

Gross appearance of HG-ESS may show a polypoid intracavitary mass or intramural mass, poorly circumscribed with myometrial invasion and cut section may reveal extensive areas of hemorrhage and necrosis. Microscopically, the tumor consists predominant high grade round, epithelloid cells with scant or moderate amounts of eosinophilic cytoplasm, containing round to oval vesicular nuclei with irregular nuclear contours and nucleoli. Tongue like myometrial invasion forming nested growth, mitotic activity of more than 10 per high power field along with the areas of necrosis is also peculiar feature of HG-ESS. ${ }^{7,8}$

Surgery including hysterectomy with salphingooophrectomy and resection of any extrauterine disease (if present) is the mainstay treatmenrt of ESS.
Complete staging and cytoreductive surgery in HG -ESS have shown to improve disease free survival (DFS). ${ }^{8}$ Though some have stated no additional advantage of oophorectomy during hysterectomy, however oophorectomy in the patients with retained ovaries have shown to improve the DFS of $100 \%$ when compared to $25 \%$ among the patients without oophorectomy. ${ }^{9}$ However it can be an option for pre-menopausal women with stage I disease. The prognostic significance of complete lymphadenectomy still remains controversial. ${ }^{8}$ Zhang et al have found that lymph node and omentum dissection play no role in prognosis. ${ }^{6}$ External pelvic irradiation has been widely used as adjuvant treatment for HS-ESS in order to decrease the recurrence;however it hasn't shown any pathbreaking result regarding the overall survival (OS) concluding that adjuvant radiotherapy may be speculated to decrease local recurrence but its impact on the overall survival is questionable. ${ }^{10}$ Use of adjuvant chemotherapy (doxorubicin, ifosphamide and cisplatin) is reasonable in the particularly aggressive disease as the recurrence pattern of HG-ESS is often distant and visceral. ${ }^{7}$ The addition of chemotherapy to radiotherapy have shown to improve 3 year survival rate and overall survival though the results did not reach statistical significance. ${ }^{10}$

Hence, the use of adjuvant radiotherapy and chemotherapy in the management of HS-ESS deserves further investigation.

\section{CONCLUSIONS}

Highgrade endometrial stromal sarcomais the rarest of the rare uterine sarcoma. Preoperative diagnosis is a challenge as the clinical and radiological features resemble to that of fibroid uterus or polyp. Complete surgical staging is the primary treatment and also important prognostic factor for disease free survival along with age at diagnosis and stage of the disease.

\section{REFERENCES}

1. Zhang Y, Chen C, Ren M, Cong X, Yang L. Treatment of uterine high grade endometrial stromal sarcoma with apatininb combined with chemotherapy. Medicine.2019;98(13):1-3. doi: 10.1097/MD.0000000000015050

2. Puliyath G, Nair VR, Swetha Singh. Endometrial stromal sarcoma.Indian J Med PaediatrOncol. 2010;31(1):21-4. DOI: 10.4103/09715851.68848 .

3. Barek JS, Hacjer NF. Barek and Hacker's gynecologic oncology. Sixth edition. WolaterKluwer.Philadelphia. 2015

4. Angelos D, Anastasios L, Dimosthenis M, Roxani D, Alexia P, Konstantinos D. Endomerial stromal sarcoma presented as endometri- 
al polyp: a rare case. Gynaecol Surg. 2020;17:6. https://doi.org/10.1186/s10397-020-01073-4

5. Eamudomkarn $\mathrm{N}$, Itarat $\mathrm{Y}$, Kleebkaow $\mathrm{P}$, Kietpeerkool C. A case report of high grade endometrial stromal sarcoma: A rare cause of abnormal uterine bleeding in a young women. Case reports in Obstet Gynecol.2018:1-2. https:// doi.org/10.1155/2018/5906760

6. Zhang YY, Li Y, Qin M, Cai Y, Jin Y, Pan LY. High-grade endometrial stromal sarcoma: a retrospective study of factors influencing prognosis. Cancer Manag Res. 2019;11:831-7.https:// doi.org/10.2147/CMAR.S187849

7. Horng HC, Wen KC, Wang PH, Chen YJ,Yen MS, Tat H. Uterine endometrial stromal sarcoma: The TAG systematic review. Taiwan J Obstet Gynecol. 2016;55(4):472-9. doi: 10.1016/j.tjog.2016.04.034
8. Agrawal R, Rajanbabu A, Nair IR, Chandramouli S, Georgeena J. Endometrial stromal sarcoma- A retrospective analysis of factors affecting recurrence. Eur J ObstetGynecolReprod Bio. 2017;216:92-7. doi: 10.1016/ j.ejogrb.2017.07.011

9. Putier P, Nam EJ, Provencher DM, Hamilton AL, Mangili G. Gynecologic cancer intergroup (GCIG) consensus review of high grade undifferentiated sarcoma of the uterus. Int J Gynecol Cancer. 2014;24(9 Suppl 3):S73-7. doi: 10.1097/IGC.0000000000000281

10. Reed NS, Malmstrom H, Scarfone G, Poveda A, PecorelliS, Tateo S et al. Phase III randomized study to evaluate the role of adjuvant pelvic radiotherapy in the treatment of stage I and II.Eur J Cancer. 2008; 44(6):808-18.doi: 10.1016/j.ejca.2008.01.019 